\title{
Recent QCD-related studies with the BABAR detector
}

\author{
Laura Zani ${ }^{* \dagger}$ \\ INFN - National Institute for Nuclear Physics \\ E-mail: laura.zani@pi.infn.it
}

\begin{abstract}
We report some of the most recent results in studying different aspects of QCD with about $500 \mathrm{fb}^{1}$ of data collected by the BABAR experiment at the $e^{+} e^{-} B$-factory PEP-II. Among these, we present a high precision measurement of the mass difference between the $D^{\star+}(2010)$ and $D^{+}$mesons using the decay chain $D^{\star+}(2010) \rightarrow D^{+} \pi^{0}$, with $D^{+} \rightarrow K^{-} \pi^{+} \pi^{+}$. This result is then combined with a previous BABAR measurement of $m\left(D^{\star+}(2010)\right)-m\left(D^{0}\right)$ to extract the mass difference between the charged and neutral $D$ mesons. We obtain results that are approximately five times more precise than the present world averages. We also report on the search for a $B$-meson decay to four baryons, $B^{0} \rightarrow p p \bar{p} \bar{p}$, which can help to shed light on the experimental discrepancy between the inclusive branching fraction of all $B$ meson decay modes with at least a couple of baryons in the final state, measured by ARGUS to be $(6.8 \pm 0.6) \%$, and the sum of exclusive baryonic channels. Finally, we present a measurement of the spectral function for the $\tau^{-} \rightarrow K^{-} K_{S} v_{\tau}$ decay, which can be used to determine the hadronic contribution to the muon $g-2$ due to the vacuum polarization.
\end{abstract}

XXVI International Workshop on Deep-Inelastic Scattering and Related Subjects (DIS2018) 16-20 April 2018

Kobe, Japan

\footnotetext{
* Speaker.

${ }^{\dagger}$ On behalf of the BABAR collaboration.
} 


\section{The data sample and the BABAR detector}

The results presented here are based on the analysis of the data collected with the BABAR detector [1] at the center of mass (CM) energy of $10.58 \mathrm{GeV}$, which corresponds to the mass of the $r(4 S)$ resonance, and $40 \mathrm{MeV}$ below. In its nine years of operation, from 1999 to 2008, the BABAR experiment was operated at the electron-positron asymmetric-energy collider PEP-II at SLAC laboratory, providing a total integrated luminosity of about $471 \mathrm{fb}^{-1}$. Charged-particle momenta are precisely measured by means of a five-layer double-sided silicon vertex tracker and a 40-layer multiwire drift chamber, both operating in the $1.5 \mathrm{~T}$ magnetic field of a superconducting solenoid. A CsI(Tl) crystal electromagnetic calorimeter measures the energy deposit of particles and photons traversing it, which is then used for the identification of charged and neutral particles. The particle identification (PID) for protons, kaons and pions uses the specific energy loss measured in the tracking devices and the measurement of the Cherenkov angle provided by the internally reflecting ring-imaging Cherenkov detector.

\section{The measurement of the $m\left(D^{\star+}(2010)\right)-m\left(D^{+}\right)$mass difference}

From the measurement of $\Delta m_{+}=m\left(D^{\star+}(2010)\right)-m\left(D^{+}\right)$, it is possible to extract the mass difference between the neutral and charged $D$ mesons $\Delta m_{D}=m\left(D^{+}\right)-m\left(D^{0}\right)$, which is a strong constraint for the calculations of symmetry breaking due to differing $u$ and $d$ quark masses and electromagnetic interactions in chiral perturbation theory [2] and lattice QCD [3] frameworks. This is done by combining the measurement of the $\Delta m_{+}$[4] with a previous $B A B A R$ measurement for the mass difference $\Delta m_{0}=m\left(D^{\star+}(2010)\right)-m\left(D^{0}\right)$ [5] and computing the $D$ mesons mass difference as $\Delta m_{D}=\Delta m_{+}-\Delta m_{0}$. The most accurate measurement of this quantity is currently provided by LHCb, $\Delta m_{D}=(4.76 \pm 0.12 \pm 0.07) \mathrm{MeV}$ [6]. However, a more precise constraint comes from the measurement of $\Delta m_{+}$by comparing the invariant mass distributions of $D^{*+}$ and $D^{+}$measured in the process $D^{*+} \rightarrow D^{+} \pi^{0}, D^{+} \rightarrow K^{-} \pi^{+} \pi^{+}$.

\subsection{The Analysis Method}

The analysis strategy consists in reconstructing the decay chain $D^{*+} \rightarrow D^{+} \pi^{0}, D^{+} \rightarrow K^{-} \pi^{+} \pi^{+}$ and extracting the mass difference $\Delta m_{+}$by fitting the distribution of the difference between the invariant masses of the reconstructed $D^{*+}$ and $D^{+}$candidates. In the $\pi^{0} \rightarrow \gamma \gamma$ reconstruction, both photon energies are required to be above $60 \mathrm{MeV}$, the total energy to be greater than 200 $\mathrm{MeV}$, and the diphoton invariant mass to be between $120 \mathrm{MeV}$ and $150 \mathrm{MeV}$. Each photon pair is kinematically fitted to the hypothesis of a $\pi^{0}$ coming from the event primary vertex and with the diphoton mass constrained to the nominal $\pi^{0}$ mass. For the $D^{+}$reconstruction, three tracks identified as kaon and pions are fitted to a common vertex and the resulting candidate is accepted only if its invariant mass is within the range $[1.86,1.88] \mathrm{GeV} / c^{2}$. Further rejection is achieved by requiring each $D^{+}$daughter track to have a polar angle measured in the laboratory frame which satisfies the condition $\left|\cos \theta_{t}\right|<0.89$. An additional selection for rejecting the peaking background is applied on the output of a likelihood variable, based on the Dalitz plot position and on the decay vertex of the candidate. Finally, the $D^{*+}$ candidate results from the combination of the previous two candidates. We reject the background contamination by performing a geometric and kinematic 
fit to both the $D^{*+}$ production vertex and the $D^{+}$decay vertex. The shape of the signal component in the mass difference distribution is studied on the simulation and fixed to the values determined from the fit to the MC sample, while the shape of the background component is determined from the fit to the data.

\subsection{The Results}

The measurement of $\Delta m_{+}$is extracted by performing an unbinned maximum likelihood fit to the data on more than 150000 signal events, which yields $\Delta m_{+}=(140597.6 \pm 6.8[\mathrm{stat}]) \mathrm{keV}$. The mass difference extracted from the fit is corrected for the bias of $3.4 \mathrm{keV}$ resulting from the peaking background, which has not been modeled in the fitting function and its contribution has been evaluated by performing pseudoexperiments. The total systematic uncertainty is $12.9 \mathrm{keV}$ and the main contribution comes from the MC-data differences in the reconstructed photon energy, due to some miscalibration of the electromagnetic calorimeter. The final result, after adding the computed bias and taking into account all the uncertainties, yields $\Delta m_{+}=(140601.0 \pm 6.8[\mathrm{stat}] \pm$ 12.9 [syst] $) \mathrm{keV}$ and combining this measurement with the previous $B A B A R$ measurement of $\Delta m_{0}=$ $(145425.9 \pm 0.5[\mathrm{stat}] \pm 1.8[\mathrm{syst}]) \mathrm{keV}$, based on the same dataset, an estimate of $\Delta m_{D}=(4824.9 \pm$ $6.8[\mathrm{stat}] \pm 12.9[\mathrm{syst}]) \mathrm{keV}$ is obtained, five times more accurate than the world average.

\section{The search for the $B^{0} \rightarrow p p \bar{p} \bar{p}$ decay}

$B$ mesons, because of their large mass, are able to decay also into final states containing baryons and are an optimal laboratory to study the mechanism of baryonic fragmentation of hadrons, only poorly understood. The inclusive branching fraction of $B$ mesons decaying into baryonic final states is approximately 7\% [7] and it is not covered by the sum of the measured exclusive baryonic channels of the $B$ meson. Indeed, this puzzle motivates the search for unmeasured $B$-meson decays to baryons. Main open issues in baryonic $B$ decays are related to the hierarchy of the branching fractions, due to resonant sub-channels, and the threshold enhancement effect. So far, the only four-baryon final state studied by the BABAR collaboration is the decay mode $\bar{B}^{0} \rightarrow \Lambda_{c}^{+} p \bar{p} \bar{p}$ [8], for which no event was observed and the upper limit on the branching fraction at $90 \% \mathrm{CL}$ was calculated to be $2.8 \times 10^{-6}$. From this measurement, for the search of $B^{0} \rightarrow p p \bar{p} \bar{p}$ [9] ${ }^{1}$ we assume as working hypothesis a branching fraction of $10^{-7}$ at the maximum, which is used for optimizing the selection.

\subsection{The Analysis Method}

The event is reconstructed combining four oppositely charged tracks identified as protons and anti-protons and kinematically fitted to a common vertex, with a fit probability larger than $0.1 \%$. A loose selection is applied to the kinematic variables $m_{\mathrm{ES}}=\sqrt{\left(E_{\text {beam }}^{*}\right)^{2}-\left(\vec{p}_{B}{ }^{*}\right)^{2}}$ and $\Delta E=E_{B}^{*}-$ $E_{\text {beam }}^{*}[10]$, related to the momentum, $\vec{p}_{B}{ }^{*}$, and the reconstructed energy, $E_{B}^{*}$, of the $B$-candidate and to the beam energy, $E_{\text {beam }}^{*}$, in the CM reference frame. The PID efficiency for protons is excellent for this analysis (>99\%) and mis-identification rates for wrongly assigning the proton identity to kaons and pions are lower than $1 \%$. Real protons coming from continuum hadronization

\footnotetext{
${ }^{1}$ The charge conjugate is always implied throughout this paper.
} 
processes $\left(e^{+} e^{-} \rightarrow q \bar{q}\right)$ are the main source of combinatorial background, which is rejected by imposing a signal-like selection on the output of a Boosted Decision Tree (BDT) algorithm, trained on four input variable distributions: $\Delta E, \cos \theta_{B}^{*}$, with $\theta_{B}^{*}$ being the flight angle of the $B$ meson in the $\mathrm{CM}$ frame, and event-shape variables, which discriminate between the spherical shape of a signal event $\left(e^{+} e^{-} \rightarrow B \bar{B}\right)$ and a jet-like $q \bar{q}$ event. This selection results in an efficiency of $21 \%$ computed on the signal MC simulation. The shape of the signal component in the $m_{\mathrm{ES}}$ distribution, modeled by a Gaussian function, is fixed to the result from the fit to the MC simulation, while the background component is modeled by means of the empirical ARGUS function, which depends on two parameters, the endpoint and the ARGUS shape. The first is determined by the kinematics and fixed to $5.289 \mathrm{GeV} / c^{2}$ in the final fit; the second is left floating and determined from the fit to the data, together with the signal and background yields.

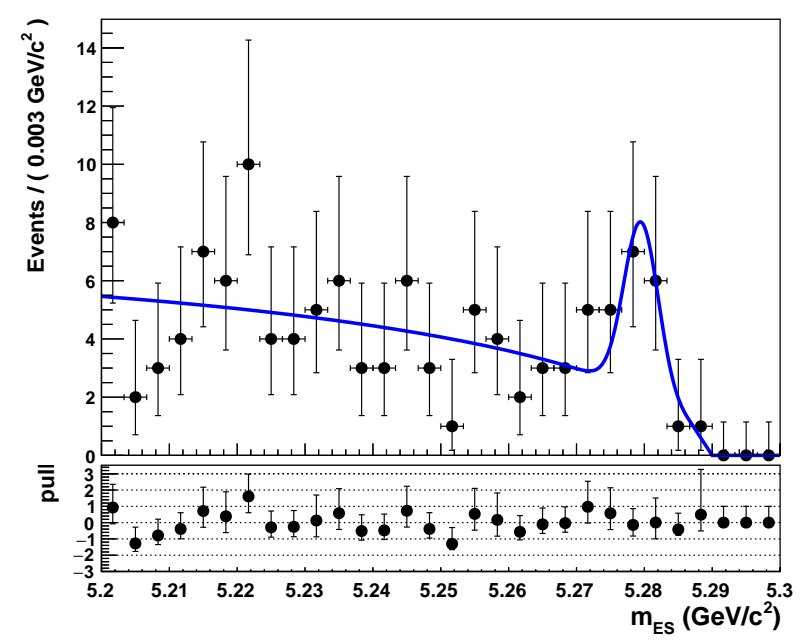

Figure 1: Preliminary result from the fit (blue line) to the data (black dots) in the whole reconstruction range $5.2<m_{\mathrm{ES}}<5.3 \mathrm{GeV} / \mathrm{c}^{2}$, after the final selection is applied. The extracted signal yield is $N_{\text {sig }}=11.1 \pm 4.6$, where the uncertainty is statistical only.

\subsection{The Results}

The signal yield is extracted from an extended unbinned maximum likelihood fit to the selected events in the range $5.2<m_{\mathrm{ES}}<5.3 \mathrm{GeV} / \mathrm{c}^{2}$ and the result is reported in Figure 1, providing a signal yield of $N_{\text {sig }}=11.1 \pm 4.6$ with a total significance of $2.9 \sigma$. The corresponding branching fraction is calculated as $\mathscr{B}\left(B^{0} \rightarrow p p \bar{p} \bar{p}\right)=N_{\text {sig }} / \varepsilon \cdot N_{B \bar{B}}=(1.14 \pm 0.47[$ stat $] \pm 0.17[\mathrm{sys}]) \times 10^{-7}$, where the experimental inputs are $N_{s i g}, \varepsilon$ and the number of $B$ meson pairs $N_{\mathrm{B} \overline{\mathrm{B}}}$. The largest contribution to the total uncertainty on the branching fraction comes from the low number of fitted signal events. The upper limit at $90 \% \mathrm{CL}$ on the branching fraction is computed in a Bayesian approach by integrating the likelihood function projected on $N_{\text {sig }}$, up to the value of $N_{\text {sig }}^{U L}$ such that the equality $\int_{0}^{N_{s i g}^{U L}} L\left(n_{s i g}\right) d n_{\text {sig }}=0.90 \int_{0}^{+\infty} L\left(n_{s i g}\right) d n_{\text {sig }}$ is verified. From an upper limit on the signal yield of $N_{\text {sig }}^{U L}=18$, we obtain $\mathscr{B}\left(B^{0} \rightarrow p p \bar{p} \bar{p}\right)<2 \times 10^{-7}$ at $90 \% \mathrm{CL}$. 


\section{The measurement of the spectral function for $\tau^{-} \rightarrow K^{-} K_{S} v_{\tau}$}

The decays of the $\tau$ lepton are useful to investigate many aspects of particle physics and the BABAR data set of more than $10^{9} \tau s$ allows very precise measurements in this field. In this analysis, the spectral function $V(q)$ for the decay $\tau^{-} \rightarrow K^{-} K_{S} v_{\tau}$ is measured, providing information about the isovector part (Isospin $=1$ ) of the total cross section for the process $e^{+} e^{-} \rightarrow K \bar{K}$ [11]:

$$
V(q)=\frac{m_{\tau}^{8}}{12 \pi C(q)\left|V_{u d}\right|^{2}} \frac{\mathscr{B}\left(\tau^{-} \rightarrow K^{-} K_{S} v_{\tau}\right)}{\mathscr{B}\left(\tau^{-} \rightarrow e^{-} v_{e} v_{\tau}\right)} \frac{1}{N} \frac{d N}{d q}
$$

with $q$ the invariant mass of the $K^{-} K_{S}$ system. The experimental inputs are the invariant mass spectrum of the $K^{-} K_{S}$ system and the branching fraction for the decay $\tau^{-} \rightarrow K^{-} K_{S} \nu_{\tau}$, whose accuracy can benefit from the large statistics available at BABAR.

\subsection{The Analysis Method}

The signal signature is defined by exploiting the event topology of $\tau$ pair events, in which one of the $\tau$ lepton decays leptonically and the other one into the signal channel. Four tracks in the event whose total charge is equal to zero are required and two oppositely charged tracks must come from the interaction point and being identified as one lepton and one kaon. To guarantee a good PID performance, the reconstructed tracks are required to be within the sensitive region of the tracking devices by imposing a selection on the measured momentum and polar angle. Furthermore, among the combinations of two charged pion tracks, only those whose invariant mass belongs to the signal region, a $25 \mathrm{MeV} / c^{2}$ large window centered on the nominal $K_{S}$ mass, are accepted. The total efficiency of the above mentioned selection, computed on the signal MC simulation, is applied in bins of $m\left(K^{-} K_{S}\right)$, resulting in an average efficiency on all the spectrum of $13 \%$. The reduction factor on background events produced by the continuum hadronization processes $e^{+} e^{-} \rightarrow q \bar{q}$ is around 5.5, while background events coming from $\tau^{+} \tau^{-}$decays are suppressed by a factor of 3.5. The remaining background is subtracted by both evaluating it on the control region in the data, which is the range in the $K_{S}$ mass spectrum complementary to the above mentioned signal region, or computing the expected contamination from MC simulations and previous measurements.

\subsection{The Results}

The resulting invariant mass spectrum of the $K^{-} K_{S}$ system, after the background subtraction, is shown in Figure 2. From the total number of selected signal events $N_{\text {sig }}=224021 \pm 3401$, the branching fraction for the decay $\tau^{-} \rightarrow K^{-} K_{S} v_{\tau}$ is computed according to the formula $\mathscr{B}\left(\tau^{-} \rightarrow\right.$ $\left.K^{-} K_{S} v_{\tau}\right)=N_{\text {sig }} /\left(L B_{\text {lep }} \sigma_{\tau \tau}\right)=(0.740 \pm 0.011[\mathrm{stat}] \pm 0.021[\mathrm{syst}]) \times 10^{-3}$, where $L=468 \pm 5 \mathrm{fb}^{-1}$ is the total luminosity, $\sigma_{\tau \tau}=0.919 \pm 0.003$ the cross section for the process $e^{+} e^{-} \rightarrow \tau^{-} \tau^{+}$at a centre of mass energy of $10.58 \mathrm{GeV}$ [12] and $B_{\text {lep }}$ the world average sum of the electronic and muonic branching fraction of the $\tau$ lepton [13]. Finally, the spectral function is computed from the measured $m\left(K^{-} K_{S}\right)$ spectrum and the above mentioned branching fraction, according to the formula in Equation (4.1). The result is reported in Figure 2 and it improves on the precision of the measurement provided by the CLEO collaboration [14]. 

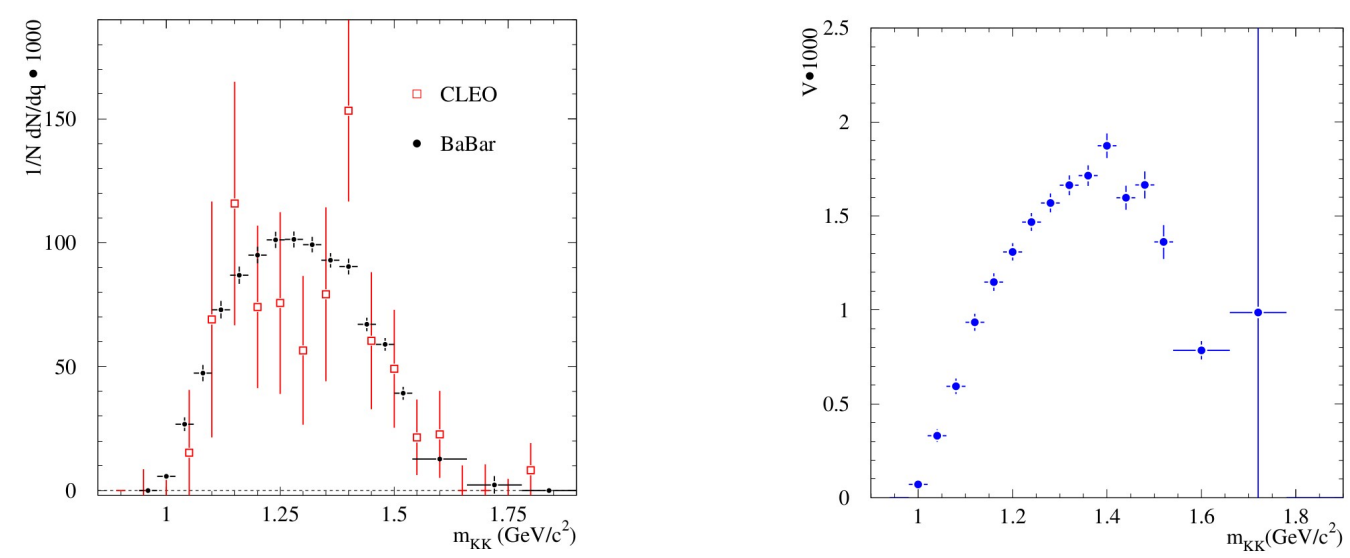

Figure 2: On the left, the normalized and efficiency corrected $m\left(K^{-} K_{S}\right)$ spectrum for the decay $\tau^{-} \rightarrow$ $K^{-} K_{S} v_{\tau}$ measured in this work (filled circles) is compared to the previous measurement by CLEO [14], performed on about 100 selected events (empty squares). On the right, the measured spectral function for the $\tau^{-} \rightarrow K^{-} K_{S} v_{\tau}$ decay is shown.

\section{References}

[1] B. Aubert et al. [BaBar Collaboration], Nucl. Instrum. Meth. A 479, 1 (2002).

[2] J. L. Goity and C. P. Jayalath, Phys. Lett. B 650, 22 (2007).

[3] R. Horsley et al. [QCDSF Collaboration], PoS Lattice 2013, 499 (2014).

[4] J. P. Lees et al. [BaBar Collaboration], Phys. Rev. Lett. 119, no. 20, 202003 (2017).

[5] J. P. Lees et al. [BaBar Collaboration], Phys. Rev. Lett. 111, no. 11, 111801 (2013).

[6] R. Aaij et al. [LHCb Collaboration], JHEP 1306, 065 (2013).

[7] Albrecht H. et al. , Zeitschrift für Physik C Particles and Fields, 56 (1992).

[8] J. P. Lees et al. [BaBar Collaboration], Phys. Rev. D 89, no. 7, 071102 (2014).

[9] J. P. Lees et al. [BaBar Collaboration], arXiv:1803.10378 [hep-ex].

[10] A. J. Bevan et al. [BaBar and Belle Collaborations], Eur. Phys. J. C 74, 3026 (2014).

[11] J. P. Lees et al. [BaBar Collaboration], Phys. Rev. D 88, no. 3, 032013 (2013).

[12] S. Jadach, B. F. L. Ward and Z. Was, Comput. Phys. Commun. 130, 260 (2000)

[13] C. Patrignani et al. [Particle Data Group], Chin. Phys. C 40, no. 10, 100001 (2016).

[14] T. E. Coan et al. [CLEO Collaboration], Phys. Rev. D 53, 6037 (1996). 